\title{
The problematic neglect of phenomenology in contemporary psychiatry
}

An adequate understanding of the phenomena of the psychoses remains elusive. This might seem a rather perplexing observation given strenuous research endeavours and a wealth of scientific information, particularly in recent years in the domains of molecular biology and neuro-imaging. A wide range of factors may be held to account for these limitations, one being that the brain is the most complex thing in the known universe, and should not be expected to yield its workings in much the same way as broken bones. Another is the enduring and profoundly mysterious gulf between observable neurobiological events and, for example, the experience of having thoughts inserted into one's brain, an enigma as much for philosophers as for psychiatrists. Related to this is a problem that some might regard as among the most significant achievements of modern psychiatry, the ICD and DSM diagnostic systems.

These systems served to bring a degree of order into the confusion of psychiatric nosology, and strove to meet, at least to some extent, the criteria of reliability and objectivity in order for psychiatry to achieve its scientific aspirations. This advance has had a number of possibly unanticipated consequences. One is that certain diagnostic categories have become reified, whereby a tentative, hypothetical construct, having perhaps a degree of validity in the light of current knowledge, is endowed with an inappropriate and confining independent status, and possibly a more doubfful validity in the light of subsequent knowledge.

Another related consequence is the relative neglect of phenomenology.' The issue is complicated by the various meanings attached to the term. Broadly, and most familiar to clinicians, is the use of the term phenomenology in a merely descriptive sense, in identifying the symptoms and signs of mental illness. Phenomenology is also used to describe a methodology, in the rigorous account of a person's singular experience of the world, and in the context of philosophical phenomenology, the investigation of the nature of experience, as opposed to the objects of experience, of not merely how the world is perceived, but of being conscious in the world. ${ }^{2}$ Clearly, within this range of meanings there is a tension between objective third-person accounts of mental states and subjective, first-person accounts, and in order to arrive at an adequate understanding of mental illness neither perspective can be disregarded.

Understanding psychotic phenomena must necessarily include the first-person account: neglecting this merely because it does not meet the criteria of scientific objectivity limits understanding and undermines scientific rigour. ${ }^{3}$ In current practice there appears to be a gulf between diagnostic explanations and a sufficient understanding of the predicament of the individual in a specific personal and social context. ${ }^{4}$ An adequate understanding of the phenomena of schizophrenia, for example, is lacking, and will require an integration of three levels: the neurobiological derangement, the aberrant cognitive processes at the psychological level, and the personal idiosyncratic experience of these processes. ${ }^{5}$ Diagnostic explanations and meaningful understanding should not be considered dichotomous, but standard clinical practice and research programmes seem to disregard the need to integrate the two perspectives, both to foster an improved therapeutic alliance and also to clarify the neurobiological basis for more disease-specific symptoms.

A further concern of the 'tick-box' psychiatry engendered by these overarching diagnostic systems is what might be considered the premature foreclosing on phenomena that require deeper levels of understanding. In the outpatient department of the public hospital where I work a beguiling gentleman with a diagnosis of schizophrenia regularly attends, although he steadfastly refuses to take medication. According to standard diagnostic criteria, and any number of rating scales, he demonstrates a formal thought disorder. Yet this description seems inadequate, and limits any further understanding of phenomena that might possibly have explanatory value. He told me: '... She took you to me ... No one wants him, she told you ... make sure you mention your own faults ...' On attending a little more closely to his utterances, it becomes evident that he is referring not to myself as the interviewer but to himself, in both the second and the third person. There is a minimal use of 'I' in his discourse; he shifts quite bewilderingly and constantly between first, second- and thirdperson perspectives. Looking beyond the surface phenomena may therefore yield potentially informative indicators of core deficits that are otherwise obscured: with regard to this instance, the shifting use of the personal pronoun suggests a disturbance of the self, or of self-consciousness, regarded by a number of commentators as the core deficit of schizophrenia. ${ }^{b}$

Yet another related concern arising from the possibly inappropriate use of the diagnostic systems is the lack of scientific validity underscoring the various diagnostic categories, the arbitrariness of operational criteria and a considerable overlap both between 
categories of mental disorders and between mental disorders and what might be considered to lie within the wide range of non-pathological human experience. This applies most obviously to problems such as anxiety and depression, but also to psychotic phenomena. The debate as to whether psychosis should be regarded as a category or a dimension has persisted for years, and again seems bound up in the tensions between validity and reliability. The argument for psychotic phenomena to be regarded as a spectrum has been given impetus by the recent attention being paid to the prevalence of psychotic experiences in the general, relatively healthy population. ${ }^{7}$ Many persons simply find ways of coping with psychotic experiences, at times creatively. Granting meaning, and embedding the phenomena in personal, social and cultural contexts, seem to be powerful ways of alleviating the distress that might otherwise ensue. Perhaps enabled by the world of Facebook, a young man in our ward told us of voices requesting to be his 'friend'. In another context, others in the same ward appear to be consoled by the belief that the voices they hear are communications from the ancestors. Merely defining these experiences as auditory hallucinations seems impoverished. Critically important information regarding both the meaning attached to the experience and the way people live with such experiences is neglected.

The issue of meaning attached to experience is of concern because it determines whether or not intervention is required, and what form of intervention might be most appropriate. Ethical dimensions of course pertain, and reflect a tension between paternalism and a more respectful, collaborative way of trying to help people living with psychotic experiences. If it is not the symptom itself but the distress or impairments it engenders, it is for the person who experiences these phenomena to decide whether or not they constitute a problem, or a symptom, and whether or not help is needed. The clinician, declaring a diagnosis of schizophrenia on the basis of hallucinations and other psychotic phenomena, might more usefully seek to understand what sense the person makes of the experiences, and to what extent they cause distress and impair functioning, as a more effective basis for further management. The assumption that psychotic experiences are inherently pathological and should therefore be suppressed needs to be reconsidered.

Another assumption underpinning the way psychosis is understood and therefore treated is the notion that the person with a psychotic disorder somehow misperceives the world 'out there', whereas the healthy general population appraises the world in a more accurate and appropriate way. This rather simplistic distinction is called into question by current neurocognitive formulations of the way the world is perceived, and enters into consciousness. This is of importance if the current notions of what might constitute schizophrenia as a disorder of consciousness are accepted. For over a hundred years it has been evident that sensory signals are relatively crude and elemental and that a time lag exists before the signals reach consciousness. Perceptions are therefore conceptualised as relatively unconscious inferences about the world. Perceptions are beliefs about the world: the notion of some sort of direct and more or less accurate perception or mirror image of an external world is an illusion. ${ }^{8}$ We all, whether psychotic or not, make up our worlds.

These possibly rather abstract considerations have implications both for management and research. If the symptoms of psychosis represent constructions, in order to cope as best as possible with what at a neurobiological level might be described as noise, these endeavours need to be acknowledged rather than merely neglected and eliminated. Modulation of salience, limiting distress and enhancing the capacity to cope might be more appropriate goals of treatment than the suppression of experiences that are desperately real for those who suffer.

The fundamental pathophysiological deficits and the ultimate causes of the syndrome of schizophrenia remain unknown. Looking beyond signs and symptoms and attending to the experience of psychosis, and linking this to observable psychological phenomena and neurobiological shifts, may yield more meaningful and productive results and more valid diagnostic constructs. First- and third-person perspectives need to be integrated if advances are to be made in the elucidation of these enigmatic phenomena. The reductive implementation of rigid scientific models has generated a bland and bleak depiction of the world of psychosis that does no justice to the extraordinary stories we are told.

\section{Sean Baumann}

Senior Specialist Psychiatrist and Lecturer Department of Psychiatry and Mental Health

University of Cape Town

1. Andreasen NC. DSM and the death of phenomenology in America: An example of unintended consequences. Schizophr Bull 2007;33(1):108-1 12.

2. Owen G, Harland R. Editor's Introduction: Theme Issue on Phenomenology and Psychiatry for the 21 st Century. Schizophr Bull 2007;33(1):105-107.

3. Strauss JS. Prognosis in schizophrenia and the role of subjectivity. Schizophr Bull 2008;34(2):201-203.

4. Van Staden W. Diagnostic understanding and diagnostic explanation in psychiatry. Curr Opin Psychiatry 2003; 16:667-671

5. Fletcher PC, Frith CD. Perceiving is believing: a Bayesian approach to explaining the positive symptoms of schizophrenia. Nat Rev Neurosci 2009; 10:48-58.

6. Cheung Chung M, Fulford KWM, Graham G, eds. Reconceiving Schizophrenia Oxford: Oxford University Press, 2007.

7. Stip E, Letourneau G. Psychotic symptoms as a continuum between normality and pathology. La Revue Canadienne de Psychiatrie 2009;54(3): 140-151. 